\title{
Effects of discrimination difficulty on the identification of different types of visual forms'
}

\author{
JOHN B. THURMOND, Performance \\ Research Laboratory, University of Louis- \\ ville, Louisville, Ky. 40208
}

The effects of different levels of discrimination difficulty on form perception were measured for two types of shapes; namely, 6 by 6 constrained metric polygons and histoforms. Five levels of discrimination difficulty were provided by use of different degrees of similarity between target and choice figures; $120 \mathrm{Ss} / 12$ in each of 10 experimental conditions) responded in a paper-and-pencil figure-cancellation task. Separate analyses of errors and response times indicated that the polygons were identified more quickly but less accurately than the histoforms. Overall performance was better with polygons than with histoforms.

Variations among shapes in the multidimensionality of form are endless (cf. Attneave \& Arnoult, 1956), and the total population of shapes to which a psychophysics of form must ultimately be generalized reaches astronomical proportions. Indeed, because shapes can differ widely in their stimulus characteristics, it may be that a particular set of derived psychophysical relations will be limited to the particular subpopulation (stimulus domain) on which they were based. Furthermore, if a reasonable degree of precision is sought, a particular psychophysical relation may be severely limitedlimited to a relatively small homogeneous population of shapes. Few comparisons, however, have been made of the performances obtained with use of equivalent forms from different types of figure populations; researchers interested in the quantification of visual form have largely employed particular classes of stimuli ranging from dot patterns (French, 1953, 1954) and bar-graph like metric histoforms (Alluisi, 1960; Baker \& Alluisi, 1962) to random polygons (Brown \& Owen, 1967; Thurmond \& Alluisi, 1967).

In the present study, identification performance was compared on two types of figures, i.e., between metric polygons (cf. Thurmond \& Alluisi, 1967) and metric histoforms at five levels of discrimination difficulty (cf. Thurmond, in press). Since these two figure types are analogs of each other, their use permits the variation and control of equated stimulus parameters. Moreover, the relatively small and homogeneous populations of metric polygons and histoforms, e.g., relative to the large and heterogeneous populations of random polygons generated by Method 1 of Attneave and Arnoult (cf. Brown \& Owen, 1967), offer a distinct advantage in measurement; namely, the psychophysical relations for each figure type can be more precisely obtained and their generalizations more cautiously limited.

Two types of figures (polygons and hist of orms) were combined factorially with five levels of stimulus difficulty to provide 10 different experimental conditions. Twelve Ss were assigned at random to each of the conditions; thus, a total of $120 \mathrm{Ss}$ served in the study.

\section{STIMULI}

Both constrained metric polygons and constrained metric histoforms were used at each of five levels of discrimination difficulty. These shapes are relatively simple, and they appear as solidly dark forms on a white background. Detailed descriptions have been given elsewhere of the bar-graph like metric histoforms (Baker \& Alluisi, 1962; Thurmond \& Alluisi, 1965) and the metric polygons (Thurmond \& Aluisi, 1967). The constrained figures were constructed by selecting the histoforms' column heights from an underlying 6 by 6 cell matrix and the polygons' radial extents from an analogous circular matrix, but with the constraint that each of the six columns (or radii) appeared once and only once in each figure. Thus, the figures represent a random sampling from two different stimulus populations, each containing 6 ! or 720 constrained metric figures. A sample of 24 constrained metric histoforms was drawn at random to serve as target figures in one figure-type condition; polygon analogs for each metric histoform were constructed to serve as target figures in the other. A restriction was imposed such that each histoform target figure differed from all others by two or more columns (and, consequently, each polygon target figure differed from all others by two or more radii). The 24 target figures in each condition were repeated in random orders for each of the five levels of difficulty. The selection of choice figures is explained later.

The metric histoforms employed as stimuli in the study were constructed of small squares (approximately 1/12 in. high) typed directly onto spirit masters with an electric typewriter. The metric polygons were accurately drawn on graph paper and transferred to spirit masters. Both types of figures were reproduced by spirit duplica- tion; the histoforms appeared as solidly dark forms and the polygons as outlined forms on one side of $8 \frac{1}{2} \times 11 \mathrm{in}$. sheets of white paper. The areas of the cell and the circular matrices were equated.

Eight of the 24 sampled target figures of a given type appeared on each of three different sheets that were stapled together to form a test representing one of the five levels of difficulty. Each of the eight problems on a page consisted of a target figure on the left, and three choice figures on the right. An example of a polygon problem at the highest level of difficulty and a hist oform problem at the lowest difficulty level is given in Figs. $1 \mathrm{~A}$ and $1 \mathrm{~B}$, respectively. The first choice figu re was $2 \frac{1 / 2}{2}$ figure widths to the right of the target, and choice figures were separated by unit figure widths. The separate problems, or rows, were separated by unit figure widths. The first- and second-choice figures were metric figures of the same type as the target, whereas the third-choice figure was an open square equal in area to the metric histoform cell matrix (1/2 in. high).

The task required $S$ to look at the target figure on the left of each row, and then to cross out either the first- or second-choice figure if it was identical to the target, or the open square if neither of the choice figures matched the target.

Each test of 24 problems called for correct responses of eight cancellations in each of the three possible choice positions, but the order was random and different for each S. The order of pages was balanced across the tests, whereas the order of target figures and correct responses was balanced within each test.

Two of the five levels of discrimination difficulty employed are illustrated in Fig. 1.

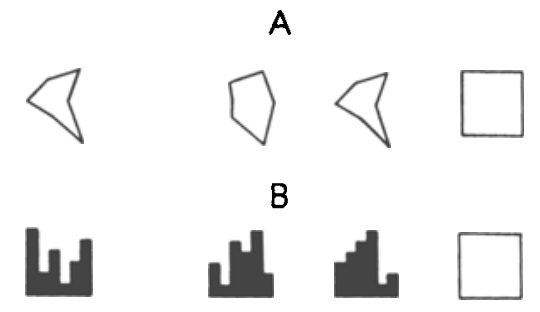

Fig. 1. Examples of metric figures and cancellation problems from 2 of the 10 experimental conditions: (A) polygonchoice figures at the highest level of discrimination difficulty, and (B) histoform-choice figures at the lowest level. 
The lowest level of discrimination difficulty, shown in Fig. 1B for the me tric histoforms, was provided by the rearrangement of all six columns between the target and choice figures (except, of course, where the choice figure was identical to the target). Since each histoform problem had a polygon analog (cf. Thurmond \& Alluisi, 1967), the lowest level of discrimination difficulty in the polygon problem was provided by the rearrangement of all six radii between the target and choice figures. It was possible to systematically increase discrimination difficulty by limiting the rearrangement of columns (or radii) between target and choice figures in successive steps from six to two (see Fig. 1B). Thus, the three intermediate levels of discrimination difficulty were obtained by use of three-, four-, and five-column (or radius) interchanges, respectively. The choice figures were constructed by selecting at random the column heights (or radial extents) to be interchanged, with the further restriction that each of the 48 choice figures ( 2 for each 24 problems) differed from each other.

\section{SUBJECTS}

The tests were administered to 120 psychology students at the University of Louisville (70 males and 50 females), 24 at each of the five levels of discrimination difficulty. They ranged in age from 16 to 65 , with a median of 21 years.

\section{PROCEDURE}

Elapsed time from the beginning of testing was written in 5 -sec intervals on a blackboard in front of the group-testing room by $\mathrm{E}$; the digits were large and clearly visible to all Ss. Each $\mathrm{S}$ recorded this time at the bottom of each page as it was completed. Thus, each of the 120 completed test booklets ( 24 at each of the five levels of difficulty) consisted of one cancellation response for each of 24 target figures, and also of three elapsed time recordings-one on each of the three sheets of the test booklet.

\section{RESULTS}

The mean time per response and the mean percentage of errors are given in Table 1 for the 10 experimental conditions of the study. ${ }^{2}$ In this table, and throughout this paper, difficulty level is coded with the numerals -2 through -6 ; this coding represents the number of column (or radius) interchanges from the highest $(-2)$ to the lowest $(-6)$ difficulty condition. With both polygons and histoforms, the two measures in Table 1 show similar trends with different difficulty levels; namely, decreases in errors and response time with decreases in the level of discrimination difficulty from -2 to -6 . Although response times for histoforms are uniformly higher than those for polygons, this measure appears to be fairly constant across levels of difficulty for both types of
Table 1

Mean Response Time per Figure Cancellation (and, in Parentheses, Mean Percentage of Errors)

\begin{tabular}{cccc}
\hline \multirow{2}{*}{$\begin{array}{c}\text { Difficulty } \\
\text { Level* }\end{array}$} & \multicolumn{2}{c}{ Type of Metric Figure } & Mean \\
\cline { 2 - 4 }-2 & Polygon & Histoform & \\
\hline \multirow{2}{*}{3} & $2.76^{* *}$ & 5.78 & 4.27 \\
-3 & $(11.50)$ & $(5.60)$ & $(8.55)$ \\
& 2.29 & 6.23 & 4.26 \\
-4 & $(10.40)$ & $(2.40)$ & $(6.40)$ \\
& 2.81 & 4.94 & 3.88 \\
-5 & $(4.20)$ & $(0.70)$ & $(2.45)$ \\
& 2.03 & 4.84 & 3.44 \\
-6 & $(2.10)$ & $(3.80)$ & $(2.95)$ \\
& 2.29 & 4.90 & 3.60 \\
& $(1.80)$ & $(1.40)$ & $(1.60)$ \\
\hline Mean & 2.44 & 5.34 & 3.89 \\
& $(6.00)$ & $(2.78)$ & $(4.39)$ \\
\hline
\end{tabular}

* Discrimination difficulty has been coded such that -2 represents the highest level (the rearrangement of only two columns, or radii, between the target and choice figures), through -6 representing the lowest level (the rearrangement of all six columns, or radii, between the target and choice figures).

** In seconds.

figures. The detrimental effects of discrimination difficulty appear to have affected identification performance more profoundly in terms of errors, especially in the case of the metric polygons.

The results ${ }^{3}$ of an analysis of variance indicated that for both errors and response times, the differences attributable to figure type were statistically significant $(F=19.06$, in the case of errors, and $F=192.68$ for response times; $\mathrm{df}=1 / 110$ and $p<.001$ in each), as were also those differences attributable to difficulty in the case of errors $(F=13.13, \mathrm{df}=4 / 110$, $p<.001)$. The interaction of these two effects was significant only in the case of the errors $(F=5.88, d f=4 / 110, p<.001)$.

\section{DISCUSSION}

It should be noted that whereas the histoforms appeared as solidly dark forms on a white background, the polygons were outlined in form; prior evidence suggest, however, that this difference in the shapes should not affect the results. Attneave (1954) has demonstrated empirically that the information in a figure is concentrated along contours and is further concentrated at the points on a contour where contour direction changes most rapidly (i.e., the angles or peaks of curvature). Thus, the assumption is not unreasonable, but the validity of this notion should be tested with further experimentation.

In the present study, a specific parameter was employed to provide a range of discrimination difficulty between target and choice figures; namely, the degree of similarity was controlled in terms of the number of column heights (histoforms) or radial extents (polygons) different in the two figures. Perceptual performance apparently differed as a function of the two different classes of shapes employed. The data of Table 1 generally indicate that the histoforms were identified less quickly but more accurately than the polygons. Other differences in performance are also apparent; thus, whereas response times were relatively constant over the levels of discrimination difficulty for both figure types, accuracy of polygon identification seems to have decreased monotonically with increases in discrimination difficulty.

These differences seem to present a paradox; that is to say, the error data indicate that metric polygons are less discriminable than metric histoforms, yet overall performance with use of polygons was higher. These differences in performance appear reasonable, however, if the data are interpreted as emphasizing the information handling aspects of visual form perception, albeit not in the sense of uncertainty analyses (cf. Baker \& Alluisi, 1962; Thurmond \& Alluisi, 1967). Indeed, stimulus uncertainty was equated in the present study by drawing samples from constrained metric-figure subpopulations of equal size. Thus, whereas the information distributed is apparently more discriminable in metric histoforms than polygons-if not phenomenally, at least as defined by accuracy of performance-the information is also processed at a slower rate.

\section{REFERENCES}

ALLUISI, E. A. On the use of information measures in studies of form perception. Perceptual \& Motor Skills, 1960, 11, 195-203.

ATTNEAVE, F. Some information aspects of visual perception. Psychological Review, 1954, 61, 183-193.

ATTNEAVE, F., \& ARNOULT, M. D. The quantitative study of shape and pattern perception. Psychological Bulletin, 1956, 53. 452-471.

BAKER, E. J., \& ALLUISI, E. A. Information handling aspects of visual and auditory form perception. Journal of Engineering Psychology, $1962,1,159-179$.

BROWN, D. R., \& OWEN, D. H. The metrics of visual form: Methodological dy spepsia. Psy chological Bulletin, 1967,68, 243-259.

FRENCH, R.S. The discrimination of dot patterns as a function of number and average separation of dots. Journal of Experimental Psychology, 1953, 46, 1-9.

FRENCH, R. S. Identification of dot patterns from memory as a function of complexity. Journal of Experimental Psy chology, 1954.47, 22-26.

LINDQUIST, E. F. Design of experiments in psychology and education. Boston: HoughtonMifflin, 1956.

ODEH, R. E., \& OLDS, E.G. Notes on the analysis of variance of logarithms of variances. USAF WADC Technical Note, 1959. No. 59-82. THURMOND, J. B. Effects of discriminability and choice-figure rotation on the visual perception of form. Joumal of Engineering Psy chology. in press. 
THURMOND, J. B., \& ALLUISI, E, A. Effects of two task variables on the visual perception of form. Joumal of Engineering Psychology, 1965. 4, 101-107.

THURMOND, J. B., \& ALLUISI, E, A. An extension of the information-deductive analy sis of form. Psychonomic Science, 1967, 7, 157-158.

\section{NOTES}

1. Supported in part by the U.S. Army Medical Research and Development Command, Department of the Army, under Contract No. DA-49. 193-MD-2567, "Behavioral Effects of Infectious Diseases." The author wishes to thank Thomas $K$ Dempsey, George W. Menzer, and Thomas J. Rebbin for technical assistance. An abridged report of this research was presented at the 60th annual meeting of the Southern Society for Philosophy and Psychology, April 1968, Louisville, Kentucky.

2. The histoform data reported here were obtained as part of a previously reported study (cf. Thurmond, in press). They are based on the responses of the $60 \mathrm{Ss}$ (12 in each of the five difficulty conditions) who were administered a nonrotated choice-figure condition first as part of a two-part test.

3. The within-cells variances of the measures were not homogeneous according to the test of homogeneity employed here and throughout the study, the technique of Odeh \& Olds (1959). Transformations of the data were not performed: rather, it was decided to rely on the characteristic robustness of the analysis of variance. This procedure was deemed necessary to avoid introducing complications of interpretation, particularly since the interaction of figure type and level of discrimination difficulty $w$ as of primary interest. Based on the Norton study of hypothetical skewed populations with unequal variances, Lindquist (1956) has concluded that even marked heterogeneity of variances produces only a negligible effect on the F-ratio distribution, and that the small effects of such heterogeneity on the probability of Type-l errors might be compensated for by the selection of a slightly smaller alpha Accordingly, Lindquist's sugge stion has been followed wherever nonhomogeneity of variance has been discovered, i.e., in these case s the $1 \%$ confidence level has been required for "statistical significant" rather than the 5 c level.

\section{Failure to replicate a reported U-shaped visual masking function}

\begin{abstract}
CHARLES W. ERIKSEN and PHILIP $H$. MARSHALL, University of Illinois, Urbana, III. 61801
\end{abstract}

An attempt was made to replicate a nonmonotonic visual backward masking function that had been reported by Kolers (1962). Neither the replication nor several variations yielded any suggestion of a nonmonotonic masking function. The failure to replicate was attributed to the difference in indicator methodology employed by Kolers and that used in the present study.

A model based upon a Rashevsky. Landahl neural net has been proposed by Weisstein (1968) to describe the temporal course of visual masking. A primary purpose of the model appears to be to describe or account for U-shaped or nonmonotonic masking functions. However, there is some real question as to whether nonmonotonic masking fucntions exist independent of artifacts arising from a confounding of masking with a delayed indicator role of the masking stimulus, indicator methodology that confuses sensitivity with aS's criterion, and/or apparent movement phenomena (Kahneman, 1967).

A major portion of the evidence for nonmonotonic masking functions comes from an experiment by Weisstein (1966). In this experiment multielement displays were employed and the masking stimulus not only served a function as a mask but also indicated to $S$ which element of the display he was to report. As Eriksen, Collins, \& Greenspon (1967) have pointed out, the resulting data represent a composite of two separate processes: a masking function per se and a function describing the decay of the icon due to the delay of the indicator telling $S$ which element to report. By appropriately manipulating constants for these two separate functions a composite curve of widely varying characteristics can be obtained.

Data reported by Kolers (1962) and Kolers \& Rosner (1960) have been widely cited as indicating nonmonotonic backward masking functions. In these studies, how. ever, a target detection or identification criterion was not employed. Instead $\mathrm{S}$ was allowed to adopt his own criterion as to the target's presence or absence and as noted elsewhere (Eriksen \& Collins, 1965), the internal consistency of the data (Kolers \& Rosner, 1960) suggests that S's criterion was extremely variable.

Nonmonotonic backward masking functions typically have not been obtained in recent masking experiments but because of their potential importance to theoretical accounts of masking it is important to determine not only whether they do exist but if they do, the specific circumstances under which they are obtained. We have been singularly unsuccessful in obtaining such functions in our own research and as a first step in an attempt to understand why, we decided to closely replicate the conditions under which other investigators have obtained these nonmonotonic functions.

One of the most pronounced nonmonotonic masking functions in the literature is given by Kolers (1962, Fig. 8) in which the target stimulus was a black-on-white dise subtending $30 \mathrm{~min}$ of angle and exposed for $50 \mathrm{msec}$. The mask was a black-on-white ring subtending $36 \cdot \mathrm{min}$ inner diam with a wall 19-min thick exposed for $100 \mathrm{msec}$ at in terstimulus intervals (ISIs) ranging from 5 to $100 \mathrm{msec}$. As reported by Kolers (1962) maximum masking occurred at an ISI value of $50 \mathrm{msec}$ where the probability of reporting the target disc was only about one-third as great as when the ISI was 5 or $100 \mathrm{msec}$. We attempted to duplicate his conditions in all essentials except that instead of using a modified quantal method (Kolers \& Rosner, 1960) we employed a two-temporal interval forced-choice indicator method. As in Kolers' experiments (Kolers, 1962; Kolers \& Rosner, 1960) viewing was monocular, but instead of always using a 3-mm artificial pupil as Kolers (1962) had done, most of our observations were collected without the artificial pupil. Pilot observations had shown that the artificial pupil did not affect the shape of the function

\section{SUBJECTS}

Six University of Illinois undergraduates, three females. served as paid volun teers. APPARATUS

AND STIMULUS MATERIALS

The stimuli were drawn with black India ink on white matte plastic cards. The discs subtended $30 \mathrm{~min}$ of visual angle. Three rings of the following dimensions were used: i.d., $36 \mathrm{~min}$, wall, $19 \mathrm{~min}$; i.d., $30 \mathrm{~min}$, wall, $25 \mathrm{~min}$; i.d., $45 \mathrm{~min}$, wall, $7 \mathrm{~min}$. A three-field Scientific Prototype Model $320 \mathrm{GA}$ tachistoscope was employed but the original equipment lamps had been replaced with Sylvania F4T5/CWX bulbs. A Macbeth illuminometer was used to calibrate and monitor intensity of the stimulus fields. The adaptation field was maintained at a luminance of $4.5 \mathrm{~mL}$ and the target and masking fields were $5.0 \mathrm{~mL}$. A black " $\mathrm{x}$ " subtending 12 min of angle, was located slightly below the center of the adaptation field and served as a fixation point. The adaptation field was illuminated at all times other than when the target or masking fields were on. The stimuli were always presented 